\title{
Alcohol solubility in a lipid bilayer: Efficient grand-canonical simulation of an interfacially active molecule
}

\author{
Jocelyn M. Rodgers, ${ }^{1, a)}$ Michael Webb, ${ }^{2}$ and Berend Smit ${ }^{3, b)}$ \\ ${ }^{1}$ Materials Science Division, Lawrence Berkeley National Laboratory, Berkeley, California 94720, USA \\ ${ }^{2}$ Department of Chemical Engineering, University of California, Berkeley, California 94720, USA \\ ${ }^{3}$ Department of Chemistry, University of California, Berkeley, California 94720, USA
}

(Received 9 November 2009; accepted 20 January 2010; published online 11 February 2010)

\begin{abstract}
We derive a new density-biased Monte Carlo technique which preserves detailed balance and improves the convergence of grand-canonical simulations of a species with a strong preference for an interfacial region as compared to the bulk. This density-biasing technique is applied to the solubility of "alcohol" molecules in a mesoscopic model of the lipid bilayer, a system which has anesthetic implications but is poorly understood. (C) 2010 American Institute of Physics.
\end{abstract}

[doi:10.1063/1.3314289]

\section{INTRODUCTION}

Herein, we present the derivation and application of a novel biased Monte Carlo (MC) approach appropriate for the adsorption of surface-active solutes at an interface. Understanding the adsorption of molecules at interfaces and accurately simulating these adsorbates in an equilibrated fashion has important consequences for a variety of processes ranging from bilayers to adsorption at solid surfaces. In this paper, we focus on the application of this MC technique to the uptake of small solutes into a lipid bilayer.

Solute adsorption at the cell membrane is an underlying biophysical component of the biological impact of alcohols and anesthetics on organisms. Meyer and Overton hypothesized a strong correlation between the oil-water partition coefficient and the anesthetic potency a century ago. ${ }^{1}$ Simulations a decade ago demonstrated an even stronger correspondence between oil-water interfacial partitioning and the action of various anesthetic molecules. ${ }^{2}$ Such partitioning into the cell membrane has consequences not only for the mechanism of general anesthesia but also for the effect of alcohol on the organisms responsible for fermentation and for the production of biofuels.

The determination of interfacial partitioning is not a straightforward process experimentally. One option, first developed by Katz and Diamond, ${ }^{4}$ involves radio labeling the solute, centrifuging the solution of lipid, solute, and water, and measuring the extent to which the solute partitions into the supernatant and the pellet. Another method, developed by Zhang and Rowe, ${ }^{5}$ involves the use of isothermal titration calorimetry to determine the bulk alcohol concentration which is in equilibrium with the alcohol solvated in the lipid bilayer vesicles. Each of these involves a certain degree of assumptions in accounting for water molecules which are directly interacting with the lipid bilayer. Other techniques shedding light on such partitioning involve drawing analogies between the partitioning coefficient and other measured

\footnotetext{
${ }^{a)}$ Electronic mail: jrodgers@berkeley.edu.

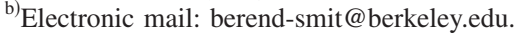

thermodynamic quantities. ${ }^{6}$ Due to the challenges associated with these various techniques, many studies of partitioning of alcohols or anesthetics into the cell membrane assume that this partitioning is independent of bulk alcohol concentration. However, alcohol solvation in water is not ideal, and solvation into the bilayer also likely introduces nonidealities due to increasingly nonlinear effects on the membrane structure as more alcohols are inserted; therefore ideality is quite a poor assumption.

Given these challenges in experimentally measuring the partitioning of solutes into the bilayer, molecular simulations could make significant contributions, both in increasing the understanding of the role of nonideality in this partitioning and also in gauging the molecular-level impact of solutes on the lipid bilayer structure. In fact, there is an extensive body of simulation work seeking to understand the effect of alcohols on the structure of the lipid bilayer. ${ }^{7-13}$

However, typical molecular dynamics simulations are ill posed for studying partitioning phenomena. To correctly sample the extent of this adsorption, solutes must make many transitions between the water and the bilayer, requiring incredibly long traditional molecular dynamics simulations.

Figure 1 illustrates these difficulties for the coarsegrained model of lipids, water, and alcohols used $;^{7}$ only very few of our model alcohols are present outside the bilayer at a mole fraction of $25 \%$ in the bilayer itself. While the solubility of actual alcohol in water is substantially higher than in our model, biophysically relevant concentrations, such as the legal limit of $0.08 \%$ blood alcohol concentration, are still quite low. Therefore transitions between bilayer and bulk will be incredibly rare within a typical simulation box. Standard grand-canonical simulations will be ineffective also because most methodologies will generate test insertions uniformly throughout the entire simulation box, but the region of likelihood and interest is explicitly at the bilayer hydrophilic-hydrophobic interface.

In this paper, we develop a density-biasing algorithm for grand-canonical MC which preserves detailed balance and correctly focuses attention for insertion in the most likely 


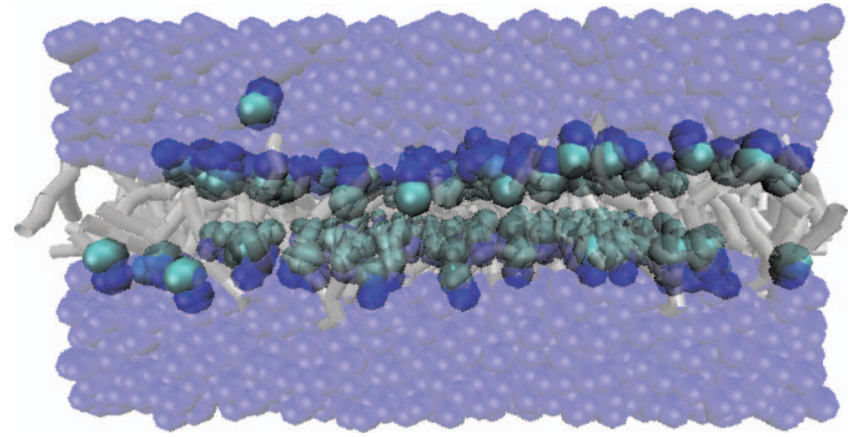

FIG. 1. Snapshot of lipid bilayer. Lipids are indicated by transparent gray, water is indicated by transparent blue, and alcohols are shown in solid blue for hydrophilic and solid turquoise for hydrophobic. The mole fraction of our model alcohol is approximately 0.25 , and even at this high mole fraction in the lipid bilayer very few molecules are in the aqueous phase.

regions of the simulation box. We explore the ability of this approach to quantitatively characterize nonideality in solute adsorption in the bilayer, the validity of our coarse-grained alcohol model in examining the concentration and temperature dependent partitioning of solutes, and finally the computational performance of this technique relative to standard grand-canonical simulations.

\section{COMPUTATIONAL DETAILS}

\section{A. Model system and simulation techniques}

We study the solubility of a short-chain alcohol in a lipid bilayer using a previously developed coarse-grained model of lipid bilayers ${ }^{14,15}$ and alcohols. ${ }^{7}$ The distance scale is set by the inclusion of three water molecules in one water bead, the cutoff distance $R_{c}$ is $6.46 \AA$ and all distances are given in reduced units of $R_{c}$. Shown in Fig. 2 are diagrams of the three molecules used, including connectivity. There exist only three types of conservative interactions in the system. First, there are short-ranged, soft pair repulsions between bead types characterized by the force (a)

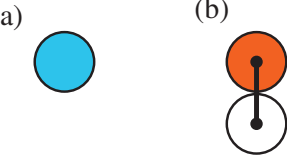

(d)

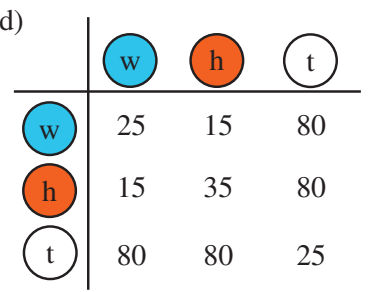

(c)

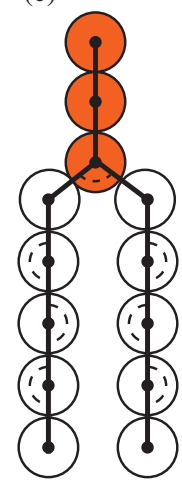

FIG. 2. Shown are the models used for simulation of (a) water, (b) ethanol, and (c) dimyristoyl phosphatidylcholine (DMPC), with the identification of the $\{\mathrm{w}\}$ water bead, the hydrophilic $\{\mathrm{h}\}$ head bead, and the hydrophobic $\{\mathrm{t}\}$ tail bead and their interactions in (d). Bonds are indicated by solid black lines connecting two bead centers. Bond-bending potentials are indicated by dashed arcs connecting to bonds.

$$
f_{i j}\left(r_{i j}\right)=a_{i j}\left(1-\frac{r_{i j}}{R_{c}}\right) \hat{r}_{i j},
$$

for $r_{i j} \leq R_{c}$. The values of $a_{i j}$ are given in Fig. 2(d) in reduced units; for the purposes of this paper, the energy units simply provide a comparative scale, but they can be qualitatively mapped back to physical temperatures and energies using membrane phase transition temperatures if desired. ${ }^{7}$

There are also two kinds of bonding potentials. Atoms connected via lines in Fig. 2 experience a bond stretching potential

$$
U_{\text {bond }}(r)=\frac{1}{2} k_{r}\left(r-r_{0}\right)^{2}
$$

with $k_{r}=100$ and $r_{0}=0.7$ in reduced units. The bond-bending terms indicated by an arc connecting two bonds have the form

$$
U_{\text {bend }}(\theta)=\frac{1}{2} k_{\theta}\left(\theta-\theta_{0}\right)^{2},
$$

with $k_{\theta}=3 \mathrm{E}_{0} / \mathrm{rad}^{2}$ for the single $\theta_{0}=90^{\circ}$ bond angle and with $k_{\theta}=6 \mathrm{E}_{0} / \mathrm{rad}^{2}$ for the remaining $\theta_{0}=180^{\circ}$ bond angles, with $\mathrm{E}_{0}$ defined as the reduced energy unit of the simulation.

This simple model of the fluid bilayer captures a substantial degree of the expected physical behavior of the true lipid bilayer, including gel, liquid-crystal, and ripple phases. ${ }^{16}$ Furthermore, alcohol models such as the one we use have been shown to induce interdigitation among the lipid tails in nonliquid crystal phases. ${ }^{7}$ We predominantly simulate the system at $T=0.7$ in order to remain in a purely liquid-crystalline phase regardless of the mole fraction of alcohol. This choice of temperature allows us to focus on the validity of our insertion method without concern for the possibility of phase separation within the bilayer induced by the addition of alcohol.

The phase space of this model is sampled in the $N_{\text {lip }} \mu_{\mathrm{A}} P_{\perp} \gamma T$-ensemble via a hybrid algorithm combining dissipative particle dynamics (DPD) trajectories with MC moves in lateral area and normal box length as well as particle insertion and deletion for the alcohol solute. The DPD trajectories evolve the coordinates of molecules, maintaining constant temperature and momentum conservation by applying random and dissipative forces on a pairwise additive basis that obey the appropriate fluctuation-dissipation relationship. The typical form for these forces is used ${ }^{14}$ in order to sample the Boltzmann distribution. ${ }^{17}$ Each individual DPD trajectory within the MC simulation had a timestep of 0.03 in reduced time units and had the number of timesteps chosen from a uniform distribution with $n_{\max }=100$ for membrane systems and $n_{\max }=50$ for bulk water systems. Within the hybrid MC-DPD simulation, DPD trajectories will compose $12 \%$ of the MC cycles, $4 \%$ of surface tension MC moves, $4 \%$ of normal pressure MC moves, and $80 \%$ of grandcanonical insertions or deletions of the cycles.

The MC moves ensure that we sample at a single thermodynamic surface tension, normal pressure, and solute chemical activity. A tensionless bilayer is maintained via MC moves. As described in previous papers, ${ }^{14}$ the constant surface tension MC move alters the lateral surface area of the lipid bilayer while maintaining a constant volume in order to do no work against external pressure. The moves are ex- 
ecuted in order to sample from the following partition function, expressing particle positions in terms of reduced positions $\mathbf{s} \equiv\left(x / L_{x}, y / L_{y}, z / L_{z}\right)$ :

$$
\begin{aligned}
Z_{N V \gamma T}= & \frac{V^{N}}{\Lambda^{3 N} N !} \frac{1}{L_{0}} \int_{0}^{\infty} d L_{y} \int d L_{z} \delta\left(L_{y}-L_{z}\right) e^{\beta \gamma L_{y} L_{z}} \\
& \times \int d L_{x} \delta\left(L_{x}-\frac{V}{L_{y} L_{z}}\right) \int d \mathbf{s}^{N} e^{-\beta U\left(\mathbf{s}^{N} ; L_{y}\right)} .
\end{aligned}
$$

A random step in the box length $L_{y}$ is chosen and $L_{z}$ is changed identically while $L_{x}$ is changed in order to maintain a constant volume. The surface tension $\gamma$ is set to zero.

We also maintain a constant pressure applied normal to the lipid bilayer via an additional MC move in order to capture the presence of a bulk water reservoir. Application of the constant normal pressure allows for varying degrees of lipid head group hydration from the water molecules while maintaining a well-defined bulk water reservoir. In reduced units, $P_{\perp}=22.28$, the pressure of bulk water at $\rho=3.0$ and $T$ $=0.32$. This temperature was chosen because it corresponds to the temperature at which the water compressibility is matched for $a_{w w}=25$ and three water molecules per bead according to the work by Groot and Warren. ${ }^{18}$ Constant pressure moves were chosen to sample from the partition function,

$$
\begin{aligned}
Z_{N P_{\perp} A T}= & \frac{1}{\Lambda^{3 N} N !} \frac{1}{V_{0}} \int_{0}^{\infty} d V V^{N} e^{-\beta p V} \\
& \times \int d L_{x} \delta\left(L_{x}-\frac{V}{A}\right) \int d \mathbf{s}^{N} e^{-\beta U\left(\mathbf{s}^{N} ; L_{x}\right)} .
\end{aligned}
$$

A random step in the box volume $V$ is chosen and then solely the simulation box length $L_{x}$ is varied. This approach ensures that no work is done relative to the surface tension during the constant pressure simulation moves. A volume change from $V_{o}$ to $V_{n}$ is accepted with the standard Metropolis acceptance ratio,

$$
\operatorname{acc}(o \rightarrow n)=\min \left[1, e^{-\beta \Delta U-\beta p \Delta V}\left(\frac{V_{n}}{V_{o}}\right)^{N}\right],
$$

where $N$ is the total number of particles in the system whose positions are scaled with the volume change. Since we hold all relative intramolecular coordinates constant during pressure and surface tensions moves, for our simulations, $N$ corresponds to the total number of molecules in the system.

In Sec. II B, we will describe how we sample the solute chemical activity in the lipid bilayer since this is based on the new density-biasing methodology. Here we describe the three different techniques used to sample the solute chemical activity in bulk water. At high chemical activities and temperatures, the solute number was varied for a set chemical activity simply via standard grand-canonical MC moves with configurational bias for the insertion of the first bead and for the tail bead. ${ }^{19}$ For these configurational bias moves, we generated 30 test configurations for the first bead and 10 test configurations for the subsequent bead.

At high temperatures but lower chemical activities, the equilibrium solute concentrations are too low to be acces- sible to such simulations. Therefore, the average solute number in a set number of solvent particles was determined using Henry's law ${ }^{19}$ written as

$$
\left\langle N_{A}\right\rangle_{\mathrm{aq}}=\frac{e^{\beta \mu}}{\Lambda^{3}}\left\langle V e^{-\beta \Delta \mathcal{U}^{+}}\right\rangle
$$

for the NPT ensemble, where $\left\langle V \exp \left(-\beta \Delta \mathcal{U}^{+}\right)\right\rangle$is determined for the insertion of a single solute particle and treated as a constant in Henry's law. Rather than using simple Widom insertion to determine $\Delta \mathcal{U}^{+}$, the excess chemical potential coupled with the volume is found via the histogram overlap of Rosenbluth factors ${ }^{20}$ for the insertion of and removal of one alcohol molecule in a bulk water simulation box of 200 beads held at constant pressure and temperature. In this instance, we confirmed that a range of number of test configurations for the Rosenbluth factors led to results identical within error bars.

Studying the temperature dependence of the solute partitioning required an alternate approach to the determination of the excess chemical potential because the histogram overlap for this dense system became progressively poorer as the temperature decreased. In such cases, we employed thermodynamic integration, ${ }^{19}$ choosing a path where the soft repulsive interactions of the alcohol beads with the solvent were scaled such that

$$
a(\lambda)=\lambda \cdot a,
$$

in order to calculate a free energy difference,

$$
\Delta F_{\text {grow }}=\int_{0}^{1} d \lambda\left\langle\frac{\partial U(\lambda)}{\partial \lambda}\right\rangle_{\lambda},
$$

where the subscript lambda indicates that the simulation was conducted with the modified $a(\lambda)$. The $\lambda$ values used for thermodynamic integration were determined via the GaussLegendre technique for approximating integrals. ${ }^{21}$ In particular, ten-point Gauss-Legendre integration was used. In this instance, again assuming Henry's law, we may express the number of solute particles solvated in 200 water molecules as

$$
\left\langle N_{A}\right\rangle_{\mathrm{aq}}=\frac{e^{\beta \mu}}{\Lambda^{3}}\langle V\rangle_{0} e^{-\beta \Delta F_{\text {grow }}},
$$

where $\langle V\rangle_{0}$ indicates the volume of the water box with no alcohol solute present.

\section{B. Density-biased Monte Carlo}

Our proposed density-biasing scheme relies on having an ansatz for the probability density $p(\mathbf{s})$ of the particles to be inserted throughout the simulation cell. We define this probability density using rescaled coordinates

$$
\mathbf{s}=\left(\frac{x}{L_{x}}, \frac{y}{L_{y}}, \frac{z}{L_{z}}\right),
$$

such that this probability density is normalized over the unit interval as 


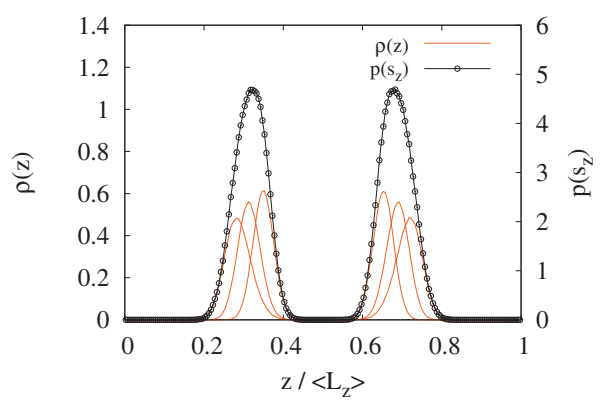

FIG. 3. The density-biasing function $p(\mathbf{s})$ as determined by the density profiles of the three lipid hydrophilic headgroup beads.

$$
\int_{0}^{1} \int_{0}^{1} \int_{0}^{1} p(\mathbf{s}) d s_{x} d s_{y} d s_{z}=1
$$

For discussion here, we estimate $p(\mathbf{s})$ for the first hydrophilic bead of the alcohol based on the density profiles of the three hydrophilic head groups in a pure lipid bilayer. This $p(\mathbf{s})$ is shown on the same plot as the density profiles associated with the three head beads in Fig. 3.

By simply choosing the z-position of the first particle in a chain from the probability distribution $p(\mathbf{s})$ rather than uniformly sampling $\mathbf{s}$, detailed balance requires

$$
\frac{\operatorname{acc}(N \rightarrow N+1)}{\operatorname{acc}(N+1 \rightarrow N)}=q(T) e^{\beta \mu} \frac{V}{N+1} \frac{\exp (-\beta \Delta \mathcal{U})}{p\left(\mathbf{s}_{1}\right)}
$$

for standard GC moves, and

$$
\frac{\operatorname{acc}(N \rightarrow N+1)}{\operatorname{acc}(N+1 \rightarrow N)}=q(T) e^{\beta \mu} \frac{V}{N+1} \frac{\mathcal{W}}{p\left(\mathbf{s}_{1}\right)}
$$

for GCMC with configurational bias moves. In these equations, $q(T)$ represents the momentum degrees of freedom for a molecule (replacing $\Lambda^{-3}$ ) and $\mathcal{W}$ is the typical normalized Rosenbluth factor used for grand-canonical MC of chain molecules. ${ }^{19}$ The full derivation of these acceptance rules based on the principle of detailed balance, both with and without configurational bias, is given in Sec. III. The function $p\left(\mathbf{s}_{1}\right)$ does not have to result from the density profile of the system provided that there is a sufficient overlap.

The crux of this technique is the use of $p(\mathbf{s})$ in the generation of trial positions for the first bead and the inclusion of this probability in the subsequent acceptance or rejection of a generated trial move. Essentially, trial move generation is biased toward the likely region of configuration space using $p(\mathbf{s})$, and this bias in move generation is removed by the division by $p(\mathbf{s})$ for insertion moves and the multiplication by $p(\mathbf{s})$ in deletion moves. Crucially, for membrane simulations, since no region of space is ever truly disallowed, $p(\mathbf{s})$ can never be exactly zero; it is just set to a very small number in the water phase and in the lipid tail region for our hydrophilic alcohol head bead as described in the Appendix. For our configurational bias moves, as in bulk water at high alcohol activities, we generate 30 test configurations for Rosenbluth sampling for the first bead and 10 test configurations for Rosenbluth sampling for the second bead.

In the results of this paper, we used two different approaches to generating the trial configurations for the second bead in the alcohol. For all results conducted at $T=0.7$ we generated the trial configurations purely based on the harmonic bond potential and thus the conservative interaction between the head and tail bead were included in the Rosenbluth factor. For the lower temperatures where acceptance ratios were correspondingly lower, we found it advantageous to generate the trial configurations using both the harmonic bonding potential and the conservative interaction between the head and tail bead.

\section{DETAILED BALANCE AND DENSITY BIASING}

The validity of the density-biased insertion and deletion algorithm hinges on whether the scheme correctly samples the probability distribution of the grand-canonical ensemble. Here we first derive the density-biased algorithm for the simple insertion and deletion of single-site particles.

The requirement of detailed balance states that the flow from a given state with $N$ particles to another state with $N+1$ particles with one particle in configuration $\Gamma$ should be equal to the flow in the opposite direction,

$$
K(N \rightarrow N+1 \mid \Gamma)=K(N+1 \mid \Gamma \rightarrow N) .
$$

These flows are a product of three contributions: the density of states $\mathcal{N}$ associated with the initial configuration as specified by statistical mechanics, the a priori probability $\alpha$ of generating a trial move, and the probability acc of accepting such a trial move. This may be expressed as

$$
\begin{aligned}
& \mathcal{N}(N) \cdot \alpha(N \rightarrow N+1 \mid \Gamma) \cdot \operatorname{acc}(N \rightarrow N+1 \mid \Gamma) \\
& \quad=\mathcal{N}(N+1 \mid \Gamma) \cdot \alpha(N+1 \mid \Gamma \rightarrow N) \cdot \operatorname{acc}(N+1 \mid \Gamma \rightarrow N) .
\end{aligned}
$$

The only modification in this case to the standard derivation of the grand-canonical insertion and deletion probabilities ${ }^{19}$ is that now

$$
\alpha(N \rightarrow N+1 \mid \Gamma)=p\left(\mathbf{s}_{\Gamma}\right),
$$

where $\mathbf{s}_{\Gamma}$ indicates the position of the particle that is inserted.

By altering the a priori probability of generating a new position, we now must unbias the insertion and deletion probabilities as

$$
\begin{aligned}
& \operatorname{acc}(N \rightarrow N+1 \mid \Gamma)=\min \left[1, \frac{e^{\beta \mu}}{\Lambda^{3}} \frac{V}{N+1} \frac{e^{-\beta \Delta \mathcal{U}}}{p\left(\mathbf{s}_{\Gamma}\right)}\right] \\
& \operatorname{acc}(N \mid \Gamma \rightarrow N-1)=\min \left[1, \frac{\Lambda^{3}}{e^{\beta \mu}} \frac{N}{V} p\left(\mathbf{s}_{\Gamma}\right) e^{-\beta \Delta \mathcal{U}}\right],
\end{aligned}
$$

where $\Delta \mathcal{U}$ refers to the change in energy for the specified process. Thus for density-biased insertion or deletion of single particles, the sole modification lies in canceling out the a priori probability of generating that bead.

For molecules, this density biasing must be coupled with configurational bias insertions of the remainder of the molecule. The crux of such approaches lies in generating $k$ trial beads at each step of the chain growth and selecting a single such bead before progressing to the next bead in the chain. Each step in chain growth has an associated Rosenbluth factor and the final acceptance probability involves the accumu- 
lated product of the Rosenbluth factor at each step. ${ }^{19}$ Thus here we determine the acceptance probability for multiple test insertions of a single bead and then justify the extension of this acceptance probability to that of a chain molecule with the first bead inserted according to a density bias.

Following a derivation of configurational biasing in the grand-canonical ensemble, ${ }^{22}$ we employ the more stringent test of superdetailed balance. This requires a balanced flow individually for each possible set of $k$ trial configurations $\{\mathbf{b}\}_{k}$, such that the position of the $N+1$ th particle is included in the set $\{\mathbf{b}\}_{k} \equiv\left\{\mathbf{b}_{\Gamma},\{\mathbf{b}\}_{k-1}\right\}$. The state flow condition may then be represented as

$$
K\left(N \rightarrow N+1 \mid\{\mathbf{b}\}_{k}\right)=K\left(N+1 \mid\{\mathbf{b}\}_{k} \rightarrow N\right) .
$$

We again write these flows in terms of three basic contributions:

$$
\begin{aligned}
& \mathcal{N}(N) \cdot \alpha\left(N \rightarrow N+1 \mid\{\mathbf{b}\}_{k}\right) \cdot \operatorname{acc}\left(N \rightarrow N+1 \mid\{\mathbf{b}\}_{k}\right) \\
& \quad=\mathcal{N}(N+1 \mid \Gamma) \cdot \alpha\left(N+1 \mid\{\mathbf{b}\}_{k} \rightarrow N\right) \cdot \operatorname{acc}\left(N+1 \mid\{\mathbf{b}\}_{k} \rightarrow N\right) .
\end{aligned}
$$

The important modifications lie in the a priori probabilities of generating a set $\{\mathbf{b}\}_{k}$ which includes $\mathbf{b}_{\Gamma}$ and choosing $\mathbf{b}_{\Gamma}$. For the insertion of a particle, this expression is

$$
\begin{aligned}
\alpha\left(N \rightarrow N+1 \mid\{\mathbf{b}\}_{k}\right) & =\left(k ! \prod_{j=1}^{k} p\left(\mathbf{s}_{j}\right) d \mathbf{s}_{j}\right) \cdot \frac{e^{-\beta u_{\mathrm{ext}}\left(\mathbf{b}_{N+1}\right)}}{\sum_{j=1}^{k} e^{-\beta u_{\mathrm{ext}}\left(\mathbf{b}_{j}\right)}} \\
& =\left(k ! \prod_{j=1}^{k} p\left(\mathbf{s}_{j}\right) d \mathbf{s}_{j}\right) \cdot \frac{e^{-\beta u_{\mathrm{ext}}\left(\mathbf{b}_{N+1}\right)}}{W_{1}} .
\end{aligned}
$$

In the formula above, the first term is the probability to generate a given set of trial configurations. The $k$ ! reflects the number of distinct orderings of configuration-generation that lead to the same overall set of configurations. The second term reflects the probability of choosing the $\mathbf{b}_{\Gamma}$ trial configuration with energy $u_{\text {ext }}$ of particle addition, given the set $\{\mathbf{b}\}_{k}$. In the second equality, we identified the denominator with the standard Rosenbluth factor $W_{1}$ for multiple test configurations of a single bead.

For the removal of the particle, we need only to generate the $k-1$ remaining trial configurations; the $\mathbf{b}_{\Gamma}$ configuration already exists and we do not need to choose that configuration. Therefore, the a priori probability is

$$
\alpha\left(N+1 \mid\{\mathbf{b}\}_{k} \rightarrow N\right)=(k-1) ! \prod_{j=1}^{k-1} p\left(\mathbf{s}_{j}\right) d \mathbf{s}_{j} .
$$

Using the definitions of $\alpha$ above and the standard grandcanonical probability density of configurations, we find

$$
\frac{\operatorname{acc}(N \rightarrow N+1)}{\operatorname{acc}(N+1 \rightarrow N)}=q(T) e^{\beta \mu} \frac{V}{N+1} \frac{W_{1}}{k \cdot p\left(\mathbf{s}_{N+1}\right)}
$$

In this formula $q(T)$ replaced $\Lambda^{-3}$ and represents the kinetic contribution of a single molecule to the partition function.

Extending this scheme to allow for the insertion or deletion of multibead molecules, we find

$$
\frac{\operatorname{acc}(N \rightarrow N+1)}{\operatorname{acc}(N+1 \rightarrow N)}=q(T) e^{\beta \mu} \frac{V}{N+1} \frac{\mathcal{W}}{p\left(\mathbf{s}_{1}\right)},
$$

where we defined a normalized Rosenbluth factor for a chain of $n$ beads as

$$
\mathcal{W} \equiv \prod_{l=1}^{n} \frac{W_{l}}{k_{l}}=\prod_{l=1}^{n}\left(\frac{1}{k_{l}} \sum_{j=1}^{k} e^{-\beta u_{\mathrm{ext}}\left(\mathbf{r}_{l}^{j}\right)}\right) .
$$

This normalized Rosenbluth factor is simply the typical Rosenbluth factor used in grand-canonical insertion and deletion of chain molecules. Understood in this sense, densitybiased insertion simply weights the possible insertions toward the physically most likely regions and then cancels out that bias when choosing whether to accept or reject that move.

\section{RESULTS AND DISCUSSION}

We now apply this density-biased insertion technique to the partitioning of solutes into the lipid bilayer. Ideal partitioning of alcohol molecules assumes that the equilibrium defined by

$$
A(\mathrm{aq}) \rightleftharpoons A(\mathrm{lip})
$$

maintains a single partitioning coefficient $K_{p}$ defined as

$$
K_{p}=\frac{[A(\mathrm{lip})]}{[A(\mathrm{aq})]},
$$

regardless of the alcohol concentration in the aqueous phase. To the extent that alcohol-alcohol interactions are important in either phase or that the alcohol molecules perturb the lipid bilayer in a nonlinear fashion, such ideality is an incredibly poor assumption. We demonstrate that careful simulations able to characterize the extent of partitioning in the aqueous and lipid bilayer phases provide a natural route to assessing the extent and ideality of solute adsorption in the lipid bilayer.

By following a procedure similar to the determination of the adsorption isotherm using grand-canonical simulations, now with density biasing, we have a technique that can accurately probe the extent of adsorption for a range of aqueous alcohol concentrations. Such an approach relies on the grand-canonical simulation of alcohol insertions into the aqueous phase and corresponding grand-canonical simulations of alcohol insertions into the lipid bilayer. By matching chemical potentials between each type of grand-canonical simulation, we know the concentration of $A(\mathrm{aq})$ which is in equilibrium with $A$ (lip), via the following connection:

$$
A(\mathrm{aq}) \rightleftharpoons A(\text { reservoir }) \rightleftharpoons A(\text { lip }) .
$$

Nonideality may (and in fact will) be present in either of these two processes. Unless the driving forces for nonideality in vapor-aqueous and in vapor-membrane partitioning are identical, nonideality in either process will lead to a nonconstant partition coefficient. In fact, given the very different nature of the aqueous environment and the membraneenvironment, it is a near certainty that such matching of driving forces will not occur and shall therefore lead to a concentration-dependent $K_{p}$. 


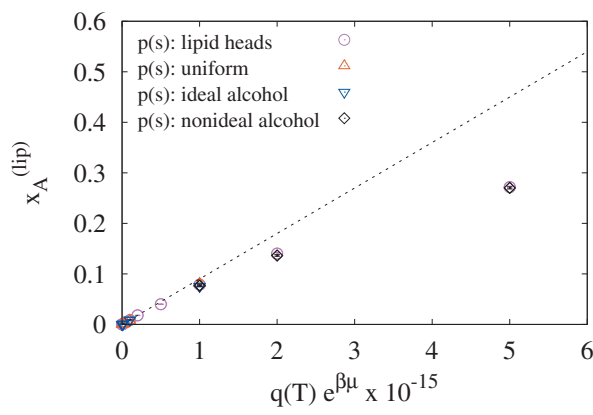

FIG. 4. Mole fraction of alcohol in the lipid phase as a function of activity $q(T) e^{\beta \mu}$. Error bars are included but smaller than the symbols. Values are displayed for several different choices of $p(\mathbf{s})$ shown in Fig. 5. The dashed line indicates the relationship dictated by Henry's law, which is only valid at low solute activities.

\section{A. Alcohol Insertion into the lipid bilayer}

We calculate the extent of alcohol solvation in the membrane as a function of the chemical activity $q(T) e^{\beta \mu}$ by applying a density bias to the trial move generation. This is achieved by simply calculating $\left\langle N_{A}\right\rangle_{\text {lip }}$ over the course of a DPD-MC trajectory. Shown in Fig. 4 is the plot of the effect of increasing chemical activity on the mole fraction of alcohol in the lipid phase, defined as

$$
x_{A}^{(\text {lip })}=\frac{\left\langle N_{A}\right\rangle_{\text {lip }}}{\left\langle N_{A}\right\rangle_{\text {lip }}+N_{\text {lip }}} .
$$

Shown in a dashed line is the result dictated by Henry's law. For small $q(T) e^{\beta \mu}$, the predicted $x_{A}^{(\text {lip) }}$ will vary linearly due to the fact that the dominant interactions are between single alcohol molecules and the surrounding lipid bilayer. Such a linear relationship corresponds to an ideal solute. As the data points deviate from Henry's law, nonideal interactions are becoming important. Physically, we may understand the decrease in $x_{A}^{\text {(lip) }}$ as a consequence of greater perturbations in the lipid bilayer. These small alcohols are interfacially active but do not contribute substantially to the overall structural integrity of the bilayer nor do their singlebead head groups contribute substantially to hydrophilic shielding of the bilayer core. Thus, as the alcohol concentration increases, the free energy penalty of further alcohol insertion becomes greater.

The mole fractions shown in Fig. 4 are determined using a variety of density biases and the results are invariant to choice of $p(\mathbf{s})$. In addition to the $p(\mathbf{s})$ based on the lipid head group density in a pure bilayer as demonstrated in Fig. 3, Fig. 5 shows the various $p(\mathbf{s})$ used. The choice of $p(\mathbf{s})=1$ is equivalent to standard configurational-bias grand-canonical MC. A more accurate choice of probability density is based on the alcohol hydrophilic density profile at an activity of $10^{14}$. This $p(\mathbf{s})$ is equivalent to the $p(\mathbf{s})$ generated from the alcohol hydrophilic bead density profile for all activity values within the region of ideality. For the higher, nonideal activities, we also examined $p(\mathbf{s})$ generated by the actual density profiles at those activities.

In order to test the effect of choice of density profile on equilibrium averages, we have run grand-canonical simulations with (i) a uniform $p(\mathbf{s})=1$, (ii) a head-group $p(\mathbf{s})$ based

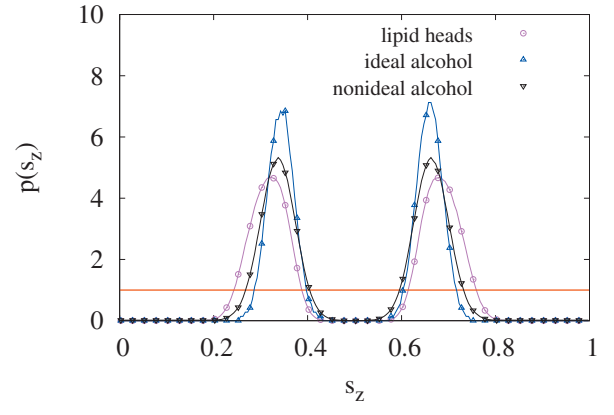

FIG. 5. Comparison of different choices of $p(\mathbf{s})$ explored in this paper. The (magenta) circles indicate $p(\mathbf{s})$ determined as shown in Fig. 3. The (blue) upright triangles indicate the $p(\mathbf{s})$ determined from the density profile of the alcohol hydrophilic bead at activity $10^{14}$, which is equivalent to the $p(\mathbf{s})$ for all activities in the ideal regime. The (black) inverted triangles show the $p(\mathbf{s})$ determined from the alcohol head bead profile specifically for the activity $5 \times 10^{15}$.

on the density profile of all hydrophilic beads in the lipids, (iii) an ideal $p(\mathbf{s})$ based on the density of the alcohol hydrophilic bead at $q(T) e^{\beta \mu}=10^{14}$, and (iv) nonideal $p(\mathbf{s})$ based on the actual densities of the alcohol hydrophilic bead at the different nonideal activities $q(T) e^{\beta \mu}=\left\{10^{15}, 2 \times 10^{15}, 5\right.$ $\left.\times 10^{15}\right\}$. The results were shown on a linear scale in Fig. 4 and are shown in Fig. 6 in logscale to emphasize the agreement over the full span of chemical activities studied. As is evident, the results are invariant for choice of $p(\mathbf{s})$. The sole substantial difference is the size of the error bars based on the quality of estimation of $p(\mathbf{s})$.

\section{B. Alcohol Insertion in the bulk}

In agreement with the conclusions of Pool and Bolhuis, ${ }^{23}$ we find that the aqueous solubilities of these soft-core repulsive amphipilic molecules is quite low. Therefore, determining the corresponding aqueous alcohol concentration proved computationally challenging. Furthermore, at chemical activities where $\left\langle N_{A}\right\rangle_{\text {aq }}$ is measurable, nonideality quickly became an important factor. As an example of this, a trace of $N_{A}$ over MC cycles is shown in Fig. 7 for two chemical activities. For $q(T) e^{\beta \mu}=10^{15}$, the number of water insertions is barely measurable over a lengthy simulation. For the higher activity, there still is relatively poor sampling, but alcohol insertions occur in bursts. These bursts in conjunc-

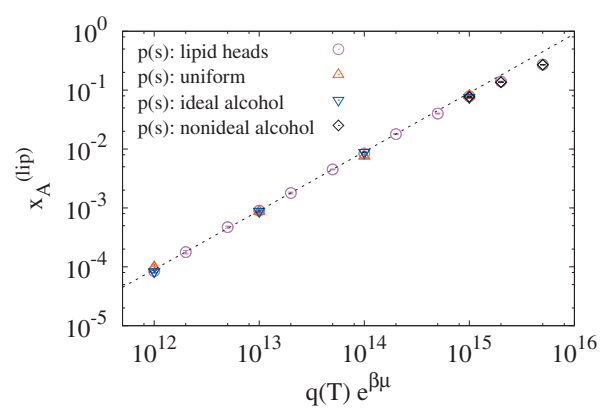

FIG. 6. Mole fraction of alcohol in lipid phase for different biasing distributions. Error bars are included for all data points but are smaller than the symbol in some cases. This plot shows the same data as Fig. 4 using a logarithmic scale in order to emphasize the agreement resulting from different density-biasing distributions across the full range of chemical activities. 


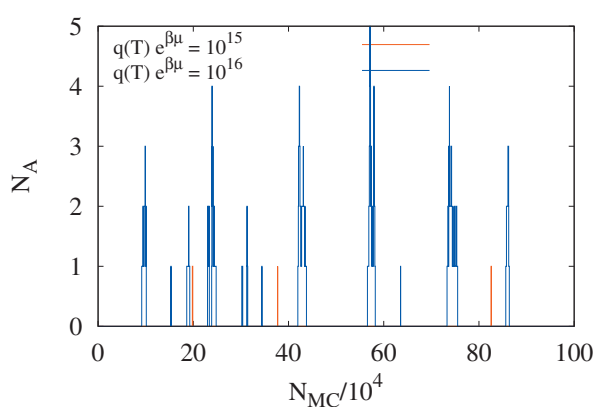

FIG. 7. Traces of number of alcohol particles in a simulation box containing 200 water beads for two different chemical activities. The transition from nearly immeasurable $\left\langle N_{A}\right\rangle$ to systems with nonideal interactions occurs quite rapidly.

tion with the observation that the alcohols tend to aggregate during these bursts suggest that we surpassed the critical micellar concentration for our model alcohol at these relatively modest concentrations. This is a consequence of the fact that our model "alcohol" can also serve as a model surfactant in general and the very low solubility of the tail beads in water. Such considerations are not immediately transferable to all alcohol models since the model we are studying was not parametrized to alcohols and ethanol in particular is fully miscible in water.

Due to the difficulty in calculating mole fractions in the aqueous phase at the relevant chemical activities, we employ two different techniques for determining

$$
x_{A}^{(\mathrm{aq})}=\frac{\left\langle N_{A}\right\rangle_{\mathrm{aq}}}{\left\langle N_{A}\right\rangle_{\mathrm{aq}}+N_{\mathrm{w}}} .
$$

At the higher chemical activities, we calculate $\left\langle N_{A}\right\rangle_{\mathrm{aq}}$ directly from simulation, and at lower chemical activities we apply Henry's law based on a chemical activity determined from histogram overlap of the Rosenbluth factors. ${ }^{20}$ Simple Widom insertion will not suffice because the average is highly dominated by the rare low energy insertion conformations. In Henry's law regime, we determine $\left\langle N_{A}\right\rangle_{\mathrm{aq}}$ using $\left\langle N_{A}\right\rangle_{\mathrm{aq}}$ $=A \cdot q(T) e^{\beta \mu}$ as described in Sec. II B with $K_{H}=3.48 \pm 0.40$ $\times 10^{-18}$.

As shown in Fig. 8, the mole fraction moves away from ideality as well at approximately the same chemical activity as nonideality appeared in the membrane system. This similarity is likely a coincidence since the driving force for non-

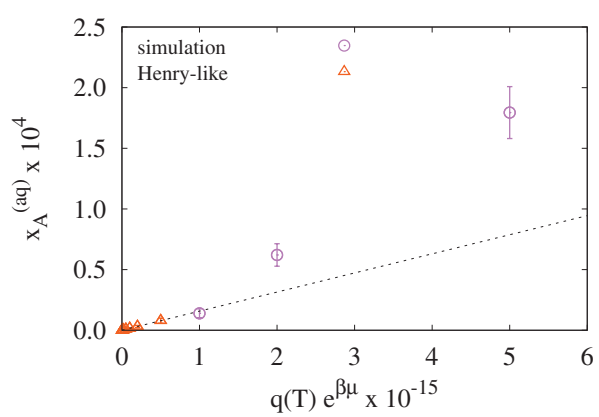

FIG. 8. Mole fraction of alcohol molecules in the aqueous phase with error bars. Those values determined from grand-canonical simulations are shown with circles, and those values determined from histogram overlap techniques and assuming Henry-like ideality are shown with triangles.

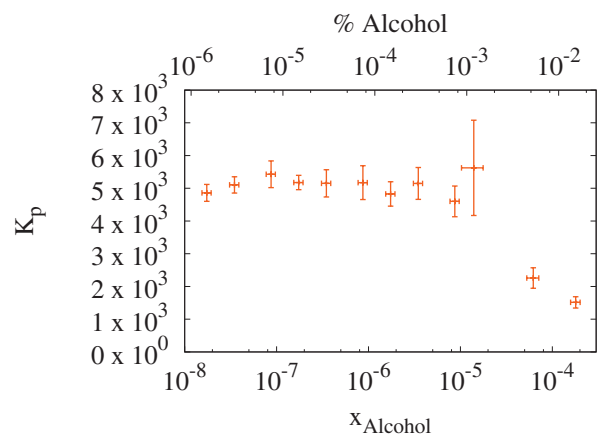

FIG. 9. Partition coefficient $K_{p}$ as a function of alcohol mole fraction with error bars. An ideal regime at small $x_{A}^{(\mathrm{aq})}$ is evident prior to a decrease in the partitioning coefficient. To give a sense of the physical concentrations, the corresponding weight percent of ethanol is given on the upper- $x$ axis, accounting for three water molecules to one water bead and assuming that the weight of the solution is essentially that of the water.

ideality in the membrane system is due to bilayer perturbation and the mechanism of solvation in the aqueous phase is quite different. The nonideality also increases the mole fraction in the aqueous phase with rising chemical potential as is experimentally observed. ${ }^{24}$ While we do not expect quantitative agreement for the onset of nonideality for alcohol solvation, our simple model for alcohols and water does capture the correct qualitative trend for the deviations from Henry's law regime, suggesting that we captured some of the essential driving forces in this system.

\section{Partition coefficient}

Given the adsorption of the alcohol into the lipid bilayer and the solvation of alcohol into the aqueous phase over a range of chemical activities, determination of the partition coefficient as a function of bulk alcohol concentration is relatively straightforward. Defining $K_{p}$ as

$$
K_{p}=\frac{x_{A}^{(\mathrm{lip})}}{x_{A}^{(\mathrm{aq})}},
$$

we find the trends in $K_{p}$ shown in Fig. 9.

As shown, $K_{p}$ remains constant up to an $x_{A}^{(\mathrm{aq})}$ of $10^{-5}$ and subsequently decreases, due to complementary deviations from Henry's law regime for both aqueous and lipid-bilayer solvation. As noted in Sec. IV B, qualitatively the deviations for alcohol in water follow the experimental trends. Furthermore, our alcohol-lipid bilayer model captures important physics related to the perturbation of the bilayer structure due to alcohol, leading to a decrease relative to Henry's law. Certainly the model for both systems neglects specific polar and hydrogen bonding interactions; therefore drawing quantitative conclusions seems ill advised. As one example of this, we know that $K_{p}$ is certainly overestimated due to the unphysically low solubility of our model alcohol in water.

Due to this the aqueous concentration for onset of nonideality is potentially misestimated as well. In order to give physical units to the mole fractions we do observe, the corresponding weight percent of alcohol is shown on the upper $y$-axis of Fig. 9. For our model, substantial nonideality is present in the biophysical range of concentrations - the full range of concentrations studied. Previous NPT simulations 


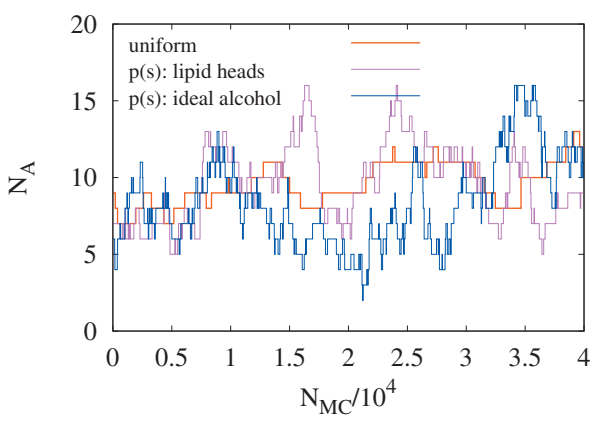

FIG. 10. Trace of $N_{A}$ in lipid bilayer for different $p(\mathbf{s})$. The variation in $N_{A}$ is substantially greater for any $p(\mathbf{s})$ which reflects the nonuniformity of the system.

found a nonideality onset as the aqueous concentration of alcohol decreased, but making quantitative statements about the nature of this nonideality and the extent of it at biophysical alcohol concentration was essentially inaccessible. ${ }^{25}$

While quantitative conclusions about actual alcoholwater-membrane systems are dubious for our chosen model system, the ability of this technique to quantitatively capture this nonideal partitioning for a given lipid bilayer and alcohol model is of note. Models which more accurately capture the molecular interactions between alcohols and lipids and alcohols and water could be treated with this approach to obtain quantitative curves of $K_{p}$ as a function of [A(aq)].

\section{Computational efficiency}

Use of the density-biased grand-canonical MC is also substantially more computationally efficient then using standard grand-canonical simulations. In Fig. 10, a trace of $N_{A}$ over the course of GCMC simulations at activity $10^{14}$ with different density-biasing qualitatively demonstrates that the traditional uniform biasing far less effectively samples the relevant numbers of alcohol particles in the membrane phases.

The percentage of GCMC insertions and deletions actually accepted indicates the frequency of sampling of different $N_{A}$ states and is therefore a direct reflection of the computational efficiency of various density biases. Shown in Fig. 11 is a plot of this acceptance rate for a variety of simulations run at an activity of $10^{14}$, on a log scale. Applying intelligent density-biasing improves the sampling of $N_{A}$ substantially. Furthermore, while the equilibrated results displayed for $x_{A}^{(\text {lip) }}$

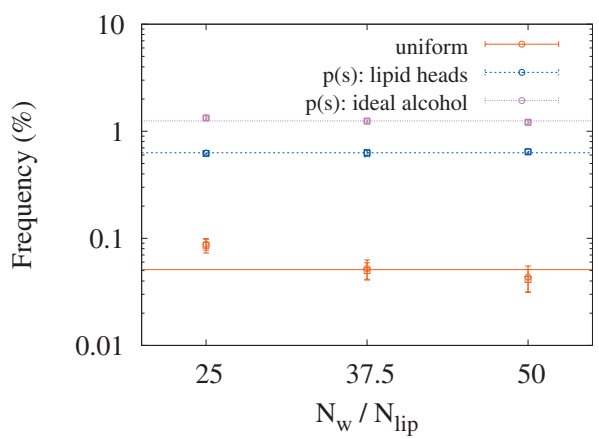

FIG. 11. Plot of sampling efficiency with different biases and different water layer widths.

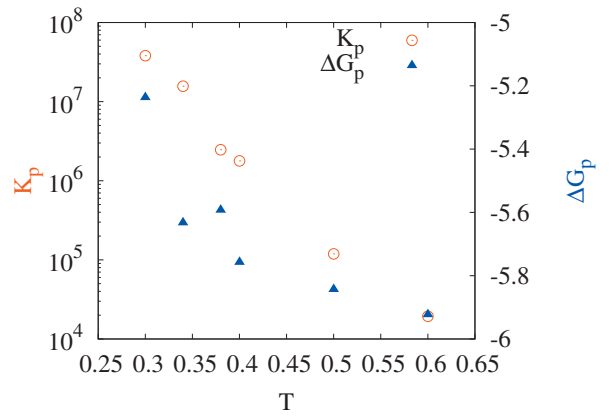

FIG. 12. Plot of $K_{p}$ for $h t$ in $h_{3}\left(t_{4}\right)_{2}$ as a function of temperature. A substantial spike in $K_{p}$ (red circles) is present, though the value at $T=0.30$ is only a lower bound on $K_{p}$. The full range is made visible via a logarithmic scale. This enhancement in $K_{p}$ however does not translate into a dip in free energy (blue triangles), where the free energy accounts for the vast range in temperatures in the system's energy units.

are independent of biasing choice, these acceptance rates make clear that the more physically informed the densitybiasing function is, the more efficiently $N_{A}$ is sampled.

Furthermore, Fig. 11 makes clear that density-biasing scales as expected with increasing simulation box volume normal to the interfacial region. Use of density-biasing functions results in an acceptance frequency that is invariant with $N_{\mathrm{w}} / N_{\text {lip }}$, whereas traditional GCMC insertion and deletion acceptance frequencies decline substantially as the water layer thickens relative to the bilayer.

\section{E. Temperature-dependence of $K_{p}$}

Experimentally, researchers observed that for small alcohols, $K_{p}$ is substantially enhanced as the temperature was lowered from the liquid crystalline $L_{\alpha}$-phase toward the ripple phase, ${ }^{6}$ but moderate length alcohols like hexanol do not exhibit this partitioning increase. ${ }^{26}$ The coarse-grained model studied undergoes the relevant phase transitions, ${ }^{16}$ and the "alcohol" model used has been shown to induce reasonable structure modification in the bilayer. ${ }^{7}$ Therefore, we studied the temperature dependence of $K_{p}$ in our model in the hope of gaining insight into the structural drivers for this increase in partitioning. For this study, the lipid model $h_{3}\left(t_{4}\right)_{2}$ was used instead of $h_{3}\left(t_{5}\right)_{2}$ since that model would have been appropriate for looking at the effects due to the presence of cholesterol as well. ${ }^{27}$

The frequency of acceptance of insertions and deletions fell substantially as $T$ dropped; therefore the trial configuration generation was modified to include the repulsive interaction between the head and tail bead in the alcohol, in order to enhance this sampling frequency. By altering the thermodynamic integration to reflect this change, Henry's constant for the corresponding reservoir was determined. While the equilibria between the reservoir and each of the two simulation boxes (pure water and lipid bilayer) were altered, the equilibrium between the aqueous and lipid phases was unaltered, as was confirmed for the higher temperatures where both trial generation approaches were employed. All simulations were conducted at the low concentration limit where Henry's law is valid.

The resulting $K_{p}$ as a function of $T$ is shown in Fig. 12. The point at $T=0.30$ is a lower bound on $K_{p}$ due to a con- 
tinually increasing $N_{A}^{(\mathrm{lip})}$ and incredibly slow equilibration. As seen, $K_{p}$ substantially increases as $T$ drops toward the main transition temperature, $T=0.30$, in our model. However the temperatures lower than the main transition temperature were computationally inaccessible. Furthermore, neither increasing alcohol tail length to species $h t_{2}$ or $h t_{3}$, altering the alcohol-head/lipid-head interactions, nor shortening the bond length within $h t$ did anything to alter this basic trend, despite reasonable hypotheses to the contrary.

By relating $K_{p}$ to a pseudo-free-energy via the transformation

$$
\Delta G_{p}=-k_{B} T \ln K_{p},
$$

we observed in Fig. 12 that the free energy of partitioning remained relatively flat. The enhancing of $K_{p}$ was rather driven by the unphysically large factor of 2 difference between $T=0.60$ and $T=0.30$ for the temperature scale in the model system. In contrast, the corresponding factor in physical units would be 1.10. Based on this, we conclude that comparing energetics and partitioning as a function of $T$ will lead to stark inaccuracies. Furthermore, based on the $\Delta G_{p}$ profile, the simple coarse-grained model used does not capture the necessary physics to explain the spike in $K_{p}$ as the main transition temperature is approached.

\section{CONCLUSIONS}

We presented a new simulation tool for studying the equilibration of solute particles between an interfacial region and a bulk phase. As shown, this density-biasing technique yields identical equilibrium results regardless of $p(\mathbf{s})$ form, including the traditional grand-canonical MC. However, use of density-biased MC yields enhanced sampling of solute particle insertions and deletions in the interfacial region. Furthermore, computational cost associated with these densitybiased insertions and deletions scales solely with the volume of the interfacial region, not with the total volume of the simulation box.

This density-biased grand-canonical MC technique has been applied to a biophysically relevant system-the equilibration of alcohol molecules between the aqueous bulk phase and the lipid bilayer. The study of such equilibration, in particular the examination of nonideality in such a partitioning process, has been computationally infeasible until now. Density biasing in tandem with a coarse-grained model of the lipid bilayer and water allowed us to quantitatively determine the partitioning coefficient $K_{p}$ as a function of alcohol concentration for the model studied.

This technique should be immediately and broadly applicable to interfacial systems of similar topology to the lipid bilayer, such as simple surface adsorption. The geometry of these systems all allow the use of a one-dimensional densitybiasing function and the associated simplifications in generating random numbers from such a distribution as described in Appendix. However, such an approach could be reasonably extended to more general geometries where regions of accessibility are limited in a known way. More complex density-biasing which is not pseudo-one-dimensional would require some more research into efficient ways of generating random numbers for those distributions.

The results of this study demonstrate a clear need for the development of new coarse-grained models which capture the physics of this partitioning over a range of temperatures. Given the dearth of experimental data related to the onset of nonideality and furthermore its dependence on temperature, simulations could contribute substantial understanding of the biophysical impact of small solutes on the cell membrane. We are currently exploring the use of these density-biasing techniques in tandem with the development of more molecularly specific coarse-grained models. ${ }^{28}$ Much work is yet to be done, but the combination of these approaches is intriguing. Given molecular models of alcohols or anesthetics which capture the thermodynamics of partitioning in a quantitative fashion, a role for molecular modeling not previously envisioned ${ }^{29}$ might be possible. The ability to fully characterize the equilibrium of an anesthetic molecule between water and the membrane over a range of chemical activities offered by density-biased grand-canonical MC could allow us to assay the potency of new anesthetics initially in silico. Furthermore, such a computational system would allow for a very natural simultaneous examination of the effect of such adsorbed bilayer solutes on proteins within the bilayer.

\section{ACKNOWLEDGMENTS}

This work was supported by the Laboratory Directed Research and Development Program of Lawrence Berkeley National Laboratory under the Department of Energy Contract No. DE-AC02-05CH11231.

\section{APPENDIX: GENERATION OF RANDOM NUMBERS FROM AN ARBITRARY $p(s)$}

We create an arbitrary $p(\mathbf{s})$ by simply summing the density profiles of beads of interest, for example the head beads of the lipids. We then guarantee that even the aqueous region will be hypothetically accessible by setting all low density values, especially zero values, to a small density that is at least the bulk density of alcohol at that given activity. Finally, normalization is enforced.

Generating random numbers according to a given $p(\mathbf{s})$ and furthermore knowing $p(\mathbf{s})$ exactly for a given value of $\mathbf{s}$ are crucial components to implementing the density-biased grand-canonical $\mathrm{MC}$ algorithm introduced in this paper. Without appropriate random number generation, detailed balance will not be obeyed.

We achieve this by taking the discrete steplike nature of collected density histograms quite literally and generating two functions based on these histograms, $p(\mathbf{s})$ and $P(\mathbf{s})$, where $P$ is the cumulative probability at any given position ranging from 0 to 1 monotonically. An example of these two functions are shown in Fig. 13, with very coarse $\Delta s$ chosen to better display the effect of this discretization on the form of each of these two functions.

Solely knowing $p(\mathbf{s})$ allows us generate the probability density associated with a given particle position, necessary for the removal probability of a particle. By inverting $P(s)$ 


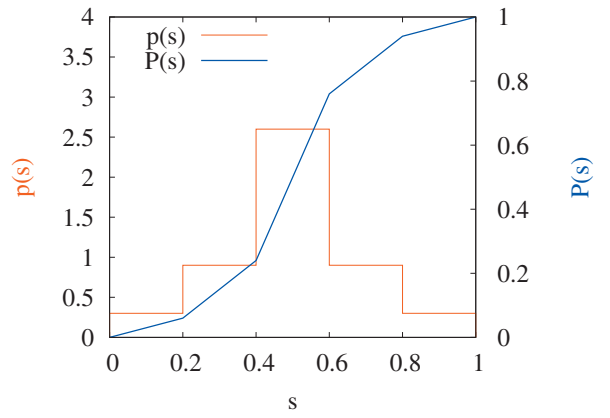

FIG. 13. Demonstration of $p(\mathbf{s})$ and $P(\mathbf{s})$ for a sample distribution which has been discretized into five regions. For density-biasing distributions, a much finer discretization of 200 points is used.

into $s(P)$, which is possible since it is a monotonic function, we may generate a random number from $p(\mathbf{s})$ simply by generating a random number $\xi$ from the uniform distribution $[0,1]$ and determining $s(\xi)$ from the inverse cumulative probability.

${ }^{1} \mathrm{~T}$. Heimburg, Thermal Biophysics of Membranes (Wiley-VCH, Germany, 2007).

${ }^{2}$ C. Chipot, M. A. Wilson, and A. Pohorille, J. Phys. Chem. B 101, 782 (1997).

${ }^{3}$ S. Liu and N. Qureshi, New Biotechnol. 26, 117 (2009).

${ }^{4}$ Y. Katz and J. M. Diamond, J. Membr. Biol. 17, 69 (1974).

${ }^{5}$ F. L. Zhang and E. S. Rowe, Biochemistry 31, 2005 (1992).

${ }^{6}$ C. Trandum, P. Westh, K. Jorgensen, and O. G. Mouritsen, Biophys. J. 78, 2486 (2000).

${ }^{7}$ M. Kranenburg, M. Vlaar, and B. Smit, Biophys. J. 87, 1596 (2004).

${ }^{8}$ A. N. Dickey and R. Faller, Biophys. J. 92, 2366 (2007).
${ }^{9}$ A. N. Dickey and R. Faller, J. Polym. Sci., Part B: Polym. Phys. 43, 1025 (2005).

${ }^{10}$ U. R. Pedersen, G. H. Peters, and P. Westh, Biophys. Chem. 125, 104 (2007).

${ }^{11}$ M. Patra, E. Salonen, E. Terama, I. Vattulainen, R. Faller, B. W. Lee, J. Holopainen, and M. Karttunen, Biophys. J. 90, 1121 (2006).

${ }^{12}$ S. Feller, C. Brown, D. Nizza, and K. Gawrisch, Biophys. J. 82, 1396 (2002).

${ }^{13}$ A. N. Dickey, W.-S. Yim, and R. Faller, J. Phys. Chem. B 113, 2388 (2009).

${ }^{14}$ M. Kranenburg, M. Venturoli, and B. Smit, J. Phys. Chem. B 107, 11491 (2003).

${ }^{15}$ M. Venturoli, M. M. Sperotto, M. Kranenburg, and B. Smit, Phys. Rep. 437, 1 (2006).

${ }^{16}$ M. Kranenburg and B. Smit, J. Phys. Chem. B 109, 6553 (2005).

${ }^{17}$ P. Español and P. Warren, Europhys. Lett. 30, 191 (1995).

${ }^{18}$ R. D. Groot and P. B. Warren, J. Chem. Phys. 107, 4423 (1997).

${ }^{19}$ D. Frenkel and B. Smit, Understanding Molecular Simulation: From Algorithms to Applications, 2nd ed. (Academic, New York, 2002).

${ }^{20}$ G. C. A. M. Mooij and D. Frenkel, J. Phys.: Condens. Matter 6, 3879 (1994).

${ }^{21}$ G. B. Arfken and H. J. Weber, Mathematical Methods for Physicists, 5th ed. (Harcourt Academic, New York, 2001).

${ }^{22}$ B. Smit, Mol. Phys. 85, 153 (1995).

${ }^{23}$ R. Pool and P. G. Bolhuis, Phys. Chem. Chem. Phys. 8, 941 (2006).

${ }^{24}$ D. A. McQuarrie and J. D. Simon, Physical Chemistry: A Molecular Approach (University Science Books, Sausalito, California, 1997), p. 979.

${ }^{25}$ E. Terama, O. H. S. Ollila, E. Salonen, A. C. Rowat, C. Trandum, P. Westh, M. Patra, M. Karttunen, and I. Vattulainen, J. Phys. Chem. B 112, 4131 (2008).

${ }^{26}$ N. Janes, J. W. Hsu, E. Rubin, and T. F. Taraschi, Biochemistry 31, 9467 (1992).

${ }^{27}$ F. de Meyer and B. Smit, Proc. Natl. Acad. Sci. U.S.A. 106, 3654 (2009).

${ }^{28}$ J. W. Chu, S. Izvekov, and G. A. Voth, Mol. Simul. 32, 211 (2006).

${ }^{29}$ J. M. Sonner, J. F. Antognini, R. C. Dutton, P. Flood, A. T. Gray, R. A. Harris, G. E. Homanics, J. Kendig, B. Orser, D. E. Raines, J. Trudell, B. Vissel, and E. I. Eger, Anesth. Analg. (Baltimore) 97, 718 (2003). 